
\title{
25 Research Square \\ The Prognostic Value of A Preoperative Lymphocyte Count and C-Reactive Protein Ratio In Osteosarcoma
}

\section{Shu-Yu Ji}

The First Affiliated Hospital of Guangxi Medical University

\section{Hai-Jun Tang}

Affiliated MinZu Hospital of Guangxi Medical University

\section{Xiao-Ting Luo}

The First Affiliated Hospital of Guangxi Medical University

\section{Wei-Feng Liang}

The First Affiliated Hospital of Guangxi Medical University

\section{Xian-Ying Huang}

First Affiliated Hospital of Guangxi University of Traditional Chinese Medicine

Shi-Jie Liao

The First Affiliated Hospital of Guangxi Medical University

\section{Wen-Yu Feng}

The First Affiliated Hospital of Guangxi Medical University

\section{Qing-Jun Wei}

The First Affiliated Hospital of Guangxi Medical University

\section{Yun Liu (D976267410@qq.com )}

The First Affiliated Hospital of Guangxi Medical University

Ju-Liang He

Guangxi Medical University Cancer Hospital

\section{Research}

Keywords: osteosarcoma, lymphocyte count and C-reactive protein ratio, prognostic nutritional index

Posted Date: January 29th, 2021

DOI: https://doi.org/10.21203/rs.3.rs-154092/v1

License: (1) (1) This work is licensed under a Creative Commons Attribution 4.0 International License. Read Full License 


\section{Abstract}

Background: Systemic inflammatory response and nutritional status are closely related to tumor development, and both have been recognized as predictors of tumors. Our study investigated the effect on the prognosis of osteosarcoma by analyzing the ratio of lymphocytes to C-reactive protein (LCR) before surgery.

Methods: Patients who were diagnosed with osteosarcoma and underwent surgery in the First Affiliated Hospital of Guangxi Medical University from 2012 to 2019 were included in this retrospective study. The albumin $(\mathrm{g} / \mathrm{L})+5 \times$ total lymphocyte count $(\mathrm{PNI})$, neutrophil/lymphocyte count (NLR), platelet/lymphocyte count (PLR) and platelet $\times$ neutrophil/lymphocyte count (SII) were calculated from preoperative peripheral white blood cells, C-reactive protein and serum albumin. The optimal cutoff values of LCR, PNI, NLR, PLR and SIl were determined by receiver operating characteristic (ROC) analysis. According to the Optimal cutoff values, LCR, PNI, NLR, PLR and SIl were divided into high and low groups. The Kaplan-Meier method was used to compare the overall survival (OS) between the high and low LCR groups. Univariate analysis was used to determine the influence of age, gender, tumor size, Enneking stage and neoadjuvant chemotherapy on the prognosis of osteosarcoma.

The independent predictors of OS were determined by Cox multivariate analysis.

Results: The optimal cutoff values for LCR, PNI, NLR, PLR and SII were 0.093, 48.4, 1.23, 157.03 and 314.27, respectively. A low preoperative LCR was significantly correlated with tumor metastasis, stage, NLR, PLR and SIl. However, a low preoperative PNI was significantly associated with tumor metastasis, stage, and PLR.

Kaplan-Meier survival analysis indicated that the postoperative OS was significantly correlated with preoperative LCR and PNI $(P<0.05)$. Univariate analysis showed that Enneking stage, metastasis and preoperative LCR, PNI, NLR, PLR and SIl were important factors affecting OS $(P<0.05)$. For multivariate analysis, the results revealed that the preoperative LCR $(H R, 0.401 ; 95 \% \mathrm{Cl}, 0.199-0.807 ; \mathrm{P}=0.01)$ and Enneking stage $(\mathrm{HR}, 2.717 ; 95 \% \mathrm{Cl}, 1.067-6.919 ; \mathrm{P}=0.036)$ is an independent prognostic factor affecting the postoperative OS of osteosarcoma.

Conclusions: The high preoperative LCR is strongly associated with longer survival time in patients with osteosarcoma. Enneking stage and preoperative LCR may be important parameters for the prognosis of osteosarcoma.

\section{Introduction}

Osteosarcoma is a common malignant bone tumors, mainly occurring in children and adolescents, with an annual incidence of approximately 4/1000000, accounting for approximately $45 \%$ of all bone and joint tumors ${ }^{[1]}$. Although surgery combined with chemotherapy and other multidisciplinary therapies can significantly improve the prognosis of patients compared with that of the previous treatments alone, The 
5-year survival rate for patients with osteosarcoma is still less than $70 \%$. The latest study found that the prognostic factors of osteosarcoma include tumor grade, histological subtypes, metastasis, alkaline phosphatase and IncRNAs, tp73-as1, circRNAs, calcitonin 3 (CNN3) and other proteins ${ }^{[2,3]}$. Although these factors have some advantages in predicting the prognosis of osteosarcoma, they also have some disadvantages, such as a complicated process, expensive price or being difficult to monitor continuously. Therefore, finding effective prognostic indicators for patients with osteosarcoma has been the focus of researchers in recent years.

The systemic inflammatory response has been recognized as the seventh characteristic of cancer ${ }^{[4]}$. Inflammatory cells and inflammatory factors widely exist in the microenvironment of osteosarcoma, which can induce the expression of various cytokines and chemokines, promote the proliferation of tumor cells and induce angiogenesis, and change the body's response to hormones and chemotherapy drugs, thus promoting the development, metastasis and drug resistance of tumors ${ }^{[5]}$.Taking the relationship between inflammation and tumor into consideration, in recent years, more and more studies have found that inflammation-related markers are prognostic factors for colorectal cancer, gastric cancer, pancreatic cancer and other malignant tumors ${ }^{[6-9]}$. Recently, some scholars have found that inflammatory indicators such as NLR, PLR and SII are correlated with the prognosis of osteosarcoma. However, there are still few reports on the influence of preoperative a LCR on the prognosis of patients with osteosarcoma. Therefore, in this study, preoperative LCR and PNI were analyzed in patients with osteosarcoma to investigate the effect of preoperative LCR on the prognosis of patients.

\section{Methods}

\section{Patients}

Patients with osteosarcoma diagnosed and operated on in the First Affiliated Hospital of Guangxi Medical University from 2012 to 2019 were included in this retrospective study. Inclusion criteria are as follows: (1) postoperative histopathological diagnosis of osteosarcoma, (2) no anticancer treatment, such as chemotherapy, radiotherapy or blood transfusion, was received before surgery, and (3) with detailed medical data and laboratory results. Exclusion criteria are as follows: (1) preoperative infection, high fever, autoimmune disease or blood system disease, (2) history of other malignant tumors, (3) receipt of nonsteroidal anti-inflammatory drugs or antibiotics, or (4) incomplete medical records. Finally, 101 patients met the above criteria to be included. Our study was conducted with the consent of Ethics Committee of Guangxi Medical University and the informed consent of each patient.

\section{Data Collection And Definition}

Through the hospital information system, we collected the gender, age, tumor size, stage, metastasis, neoadjuvant chemotherapy, pathological fracture, laboratory examination and other basic clinical data of all patients. The results of blood routine and liver function tests were obtained by drawing blood within 1 
week before the operation. The definitions of LCR, PNI, NLR, PLR and SIl were shown as follows: LCR = lymphocyte counts/ C-reactive protein; PNI = albumin $(\mathrm{g} / \mathrm{L})+5 \times$ total lymphocyte count $\left(10^{9} / \mathrm{L}\right) ; \mathrm{NLR}=$ neutrophil/lymphocyte-count; PLR = platelet/lymphocyte count; SII = platelet $\times$ neutrophil/lymphocyte count. According to receiver operating characteristic curve (ROC), the optimal truncation values of the LCR, NLR, PLR, SIl and PNI are obtained when the Youden index is the largest. LCR and PNI values greater than the corresponding cutoff values were defined as high LCR, high PNI, high NLR, high PLR, and high SII (HLCR, HPNI, HNLR, HPLR, and HSIl respectively).

\section{Follow-up}

After the operation, all patients were followed up regularly by re-examination and telephone calls. Overall survival (OS) was defined from the time of definite histopathological diagnosis until the date of last follow-up or death in this study. Follow-up was conducted every 3 months for the first 4 years, followed by every 6 months to the most recent follow-up. Follow-up included a thorough history, physical examination, laboratory tests (routine blood, blood biochemistry, tumor markers, etc.), MRI, chest CT, etc.

\section{Statistical analysis}

SPSS 24.0 Software was used for data analysis. ROC analysis was used to obtain the optimal cutoff values for continuous prognostic variables (LCR, PNI, NLR, PLR, and SII) by the maximal Youden index. The $\chi 2$ test was used to compare the differences between groups, the Kaplan-Meier was used to draw the survival curve, and the log-rank test was used to compare the survival differences between the two groups. In terms of prognostic analysis, both a univariate analysis and multivariate analysis were investigated by Cox hazard regression model. The factors were supposed to be remarkably significant. A two-tailed $p<0.05$ was considered to be statistically significant.

\section{Results}

\section{Clinical Parameters Of Patients With Osteosarcoma}

Table 1 presents the clinical parameters of the patients. Among 101 patients with osteosarcoma, 67 patients were male $(66.3 \%)$ and 34 patients were female (33.7\%). The median age was 18 years. A total of 90 tumors $(89.1 \%)$ were located in the extremities, and 11 tumors $(10.9 \%)$ were located in other parts of the body. There were 73 tumors with tumor size $\geq 5 \mathrm{~cm}$. All patients underwent surgical treatment, of which $84(83.2 \%)$ and 17 cases $(16.8 \%)$ had tumor resection and amputation, respectively. According to the Enneking stage, 62 patients (61.4\%) were in stages I and II, and 39 patients (38.6\%) were in stage III. There were 15 patients $(14.9 \%)$ with pathological fractures. Thirty patients $(29.7 \%)$ had tumor metastasis, and 21 patients $(20.8 \%)$ received neoadjuvant chemotherapy. 
Table 1

Association of the patients' clinical parameters with LCR and PNI

\begin{tabular}{|c|c|c|c|c|c|c|c|}
\hline \multicolumn{8}{|l|}{ LCR PNI } \\
\hline Variables & Cases & $\begin{array}{l}\text { Low } \\
\text { (36) }\end{array}$ & $\begin{array}{l}\text { Hight } \\
(65)\end{array}$ & $\mathrm{P}$ & $\begin{array}{l}\text { Low } \\
\text { (27) }\end{array}$ & $\begin{array}{l}\text { Hight } \\
(74)\end{array}$ & $P$ \\
\hline Gender & 101 & 25 & 42 & 0.623 & 17 & 50 & 0.665 \\
\hline Male & 67 & 11 & 23 & & 10 & 24 & \\
\hline Female & 34 & & & & & & \\
\hline Age(years) & 41 & 12 & 29 & 0.269 & 9 & 32 & 0.369 \\
\hline$<17$ & 60 & 24 & 36 & & 18 & 42 & \\
\hline \multicolumn{8}{|l|}{$\geq 17$} \\
\hline Tumor location & 90 & 33 & 57 & 0.539 & 25 & 65 & 0.497 \\
\hline Extremities & 11 & 3 & 8 & & 2 & 9 & \\
\hline \multicolumn{8}{|l|}{ Non extremities } \\
\hline Tumor size & 28 & 8 & 20 & 0.358 & 4 & 24 & 0.080 \\
\hline$<5$ & 73 & 28 & 45 & & 23 & 50 & \\
\hline \multicolumn{8}{|l|}{$\geq 5$} \\
\hline Surgical approach & 84 & 28 & 56 & 0.281 & 21 & 63 & 0.382 \\
\hline Tumor resection & 17 & 8 & 9 & & 6 & 11 & \\
\hline \multicolumn{8}{|l|}{ Amputation } \\
\hline Enneking stage & 62 & 16 & 46 & 0.009 & 12 & 50 & 0.035 \\
\hline$I+\|$ & 39 & 20 & 19 & & 15 & 24 & \\
\hline \multicolumn{8}{|l|}{ III } \\
\hline Pathological fracture & 86 & 29 & 57 & 0.334 & 24 & 62 & 0.523 \\
\hline No & 15 & 7 & 8 & & 3 & 12 & \\
\hline Yes & & & & & & & \\
\hline
\end{tabular}

Abbreviations: LCR: lymphocyte count and C-reactive protein ratio; PNI: prognostic nutritional index; NLR: neutrophil-lymphocyte ratio; PLR: platelet-lymphocyte ratio; SII: systemic immuneinflammation index. 


\begin{tabular}{|c|c|c|c|c|c|c|c|}
\hline \multicolumn{8}{|l|}{ LCR PNI } \\
\hline Local recurrence & 91 & 31 & 62 & 0.318 & 23 & 68 & 0.318 \\
\hline No & 10 & 5 & 5 & & 4 & 6 & \\
\hline \multicolumn{8}{|l|}{ Yes } \\
\hline Metastasis & 71 & 16 & 55 & 0.000 & 14 & 57 & 0.014 \\
\hline No & 30 & 20 & 10 & & 13 & 17 & \\
\hline \multicolumn{8}{|l|}{ Yes } \\
\hline Neoadjuvant & 80 & 27 & 53 & 0.438 & 19 & 61 & 0.186 \\
\hline chemotherapy & 21 & 9 & 12 & & 8 & 13 & \\
\hline \multicolumn{8}{|l|}{ No } \\
\hline \multicolumn{8}{|l|}{ Yes } \\
\hline NLR & & & & 0.019 & & & 0.058 \\
\hline$<1.23$ & 9 & 0 & 9 & & 0 & 9 & \\
\hline$\geq 1.23$ & 92 & 36 & 56 & & 27 & 65 & \\
\hline PLR & & & & $<0.001$ & & & \\
\hline$<157.03$ & 61 & 13 & 48 & & 9 & 52 & 0.001 \\
\hline$\geq 157.03$ & 40 & 23 & 17 & & 18 & 22 & \\
\hline SII & & & & 0.017 & & & 0.099 \\
\hline$<314.27$ & 18 & 2 & 16 & & 2 & 16 & \\
\hline$\geq 314.27$ & 83 & 34 & 49 & & 25 & 8 & \\
\hline $\begin{array}{l}\text { Abbreviations: LC } \\
\text { NLR: neutrophil-I } \\
\text { inflammation ind }\end{array}$ & $\begin{array}{l}\text { yte } \\
\text { ratic }\end{array}$ & Id C- & $\begin{array}{l}\text { ive p } \\
\text { ympl }\end{array}$ & $\begin{array}{l}\text { ratio; } P \\
\text { ratio; } S\end{array}$ & $\begin{array}{l}\text { ogn } \\
\text { stem }\end{array}$ & nutr & index; \\
\hline
\end{tabular}

\section{The optimal cutoff values of LCR, PNI, NLR, PLR and SII}

The death of patients with osteosarcoma was used as the endpoint. Figure 1 shows the results of ROC analysis of LCR, PNI, NLR, PLR and SII. The area under the ROC curve (AUC) of LCR and PNI was 0.727 (P $<0.001)$ and $0.671(P=0.003)$, respectively, while the AUC of NLR, PLR and SII was $0.577,0.579$ and 0.603 , respectively. The optimal cutoff value of LCR was 0.093 (sensitivity was $52.6 \%$, specificity was $86.4 \%)$, 
While the optimal cutoff value of PNI was 48.4 (sensitivity $40.4 \%$, specificity $90.9 \%$ ). The optimal cutoff value of NLR was 1.23 (sensitivity $98.2 \%$, specificity 18.2\%). The optimal cutoff value of PLR was 157.03 (sensitivity $47.4 \%$, specificity $70.5 \%$ ). The optimal cutoff value of SII was 314.27 (sensitivity $93.0 \%$, specificity $31.8 \%$ ). According to the optimal cutoff values, patients were divided into the HLCR group ( $\geq$ $0.093, n=65)$ and the low LCR group (LLCR $<0.093, n=36)$; the HPNI group $(\geq 48.4, n=74)$ and low PNI group (LPNI < 48.4, $n=27)$; the HNLR group $(\geq 1.23, n=92)$ and low NLR group $(L N L R<1.23, n=9)$; the HPLR group ( $\geq 157.03, n=40)$ and low PLR group (LPLR $<157.03, n=61) ; \operatorname{HSII}$ group $(\geq 314.27, n=83)$ and low SIl group $(<314.27, \mathrm{n}=18)$. (Table 2$)$

Table 2

ROC curve and cut-off values analysis of LCR,PNI,NLR,PLR and SII.

\begin{tabular}{|llllllll|}
\hline Variable & AUC & $95 \% \mathrm{Cl}$ & $\mathbf{P}$ & $\begin{array}{l}\text { Yoden } \\
\text { index }\end{array}$ & $\begin{array}{l}\text { Cutoff } \\
\text { values }\end{array}$ & Sensitivity & Specificity \\
\hline LCR & 0.727 & $\begin{array}{l}0.627- \\
0.826\end{array}$ & $<0.001$ & 0.390 & 0.093 & $52.6 \%$ & $86.4 \%$ \\
\hline PNI & 0.671 & $\begin{array}{l}0.567- \\
0.776\end{array}$ & 0.003 & 0.313 & 48.4 & $40.4 \%$ & $90.9 \%$ \\
\hline NLR & 0.577 & $\begin{array}{l}0.463- \\
0.691\end{array}$ & 0.186 & 0.164 & 1.23 & $98.2 \%$ & $18.2 \%$ \\
\hline PLR & 0.579 & $\begin{array}{l}0.465- \\
0.693\end{array}$ & 0.173 & 0.178 & 157.03 & $47.4 \%$ & $70.5 \%$ \\
\hline SII & 0.603 & $\begin{array}{l}0.490- \\
0.717\end{array}$ & 0.076 & 0.248 & 314.27 & $93.0 \%$ & $31.8 \%$ \\
\hline
\end{tabular}

\section{Relationship between LCR and PNI and clinical indicators of patients}

The relationship between LCR, PNI and the basic clinical features of patients with osteosarcoma is shown in Table 1. Compared with the HLCR group, the LLCR group was significantly associated with advanced Enneking stage $(P=0.009)$ and more metastasis $(P<0.001)$. However, there were no significant differences between the two groups in age, sex, pathological fracture, modality, tumor size, local recurrence or neoadjuvant chemotherapy.

In addition, the LPNI group tended to get more metastasis $(P=0.014)$ and, advanced Enneking stage $(P=$ 0.035) compare with those in the HPNI. However, there was no significant relationship between the HLCR group and LCR group in terms of internal age, sex, pathological fracture, modality, tumor size, local recurrence or neoadjuvant chemotherapy.

\section{Univariate And Multivariate Survival Analyses}

The median follow-up time of the 101 patients with osteosarcoma was 25 months (range,2-96 months), and 57 patients died. Kaplan-meier was used to analyze the difference of postoperative overall survival rate between HLCR group and LLCR group, HPNI group and LPNI group. The results suggest that the OS 
of the LLCR group was significantly lower than that of the HLCR group (median OS, 17 VS 54 months, $\mathrm{P} \otimes 0.001,95 \% \mathrm{Cl} 27.32-44.68$; Fig. 2a). Compared with the HPNI group, the OS of the LPNI group was significantly worse (median OS, 22 VS 52 months, $\mathrm{P} \otimes 0.001,95 \% \mathrm{Cl} 27.32-44.68$; Fig. 2b).

Univariate Cox regression analysis showed that tumor stage $(P<0.001)$, metastasis $(P<0.001), L C R(P<$ 0.001), $P N I(P=0.001), N L R(P=0.018), P L R(P=0.002)$ and $S I I(P<0.001)$ were significantly associated with OS. Therefore, these factors were incorporated into a Cox regression analysis for multifactor analysis. The results showed that the tumor stage $(\mathrm{HR}, 2.717 ; 95 \% \mathrm{Cl}, 1.067-6.919 ; \mathrm{P}=0.036)$ and preoperative $\mathrm{LCR}(\mathrm{HR}, 0.401 ; 95 \% \mathrm{Cl}, 0.199-0.807 ; \mathrm{P}=0.01)$ is an independent prognostic factors for OS after surgery. (Table 3 ) 
Table 3

Analysis of the factors influencing the postoperative prognosis of patients with osteosarcoma

\section{Univariate analysis Multivariate analysis}

\begin{tabular}{|c|c|c|c|c|c|c|}
\hline Variables & HR & $95 \% \mathrm{Cl}$ & $P$ & HR & $95 \% \mathrm{Cl}$ & $P$ \\
\hline Gender & 1 & $0.408-1.302$ & 0.286 & & & \\
\hline Male & 0.729 & & & & & \\
\hline \multicolumn{7}{|l|}{ Female } \\
\hline Age (years) & 1 & $0.665-2.008$ & 0.607 & & & \\
\hline$<17$ & 1.156 & & & & & \\
\hline \multicolumn{7}{|l|}{$\geq 17$} \\
\hline Tumor location & 1 & $0.654-2.949$ & 0.393 & & & \\
\hline Extremities & 1.388 & & & & & \\
\hline \multicolumn{7}{|l|}{ Nonextremities } \\
\hline Tumor size & 1 & $0.711-2.464$ & 0.377 & & & \\
\hline$<5$ & 1.323 & & & & & \\
\hline \multicolumn{7}{|l|}{$\geq 5$} \\
\hline Surgical approach & 1 & $0.456-1.795$ & 0.774 & & & \\
\hline Tumor resection & 0.904 & & & & & \\
\hline \multicolumn{7}{|l|}{ Amputation } \\
\hline Enneking stage & 1 & $1.801-5.171$ & $<0.001$ & 2.717 & $1.067-6.919$ & 0.036 \\
\hline$I+\|$ & 3.052 & & & & & \\
\hline \multicolumn{7}{|l|}{ III } \\
\hline Pathological fracture & 1 & $0.566-2.760$ & 0.581 & & & \\
\hline No & 1.250 & & & & & \\
\hline \multicolumn{7}{|l|}{ Yes } \\
\hline Local recurrence & 1 & $0.785-3.514$ & 0.185 & & & \\
\hline No & 1.661 & & & & & \\
\hline Yes & & & & & & \\
\hline
\end{tabular}




\begin{tabular}{|c|c|c|c|c|c|c|}
\hline \multicolumn{7}{|c|}{ Univariate analysis Multivariate analysis } \\
\hline Metastasis & 1 & $1.601-4.620$ & $<0.001$ & 0.703 & $0.261-1.890$ & 0.485 \\
\hline No & 2.720 & & & & & \\
\hline \multicolumn{7}{|l|}{ Yes } \\
\hline Neoadjuvant chemotherapy & 1 & $0.721-2.624$ & 0.333 & & & \\
\hline No & 1.376 & & & & & \\
\hline \multicolumn{7}{|l|}{ Yes } \\
\hline LCR & 1 & $0.153-0.449$ & $<0.001$ & 0.401 & $0.199-0.807$ & 0.01 \\
\hline Low & 0.262 & & & & & \\
\hline \multicolumn{7}{|l|}{ High } \\
\hline PNI & 1 & $0.225-0.660$ & 0.001 & 0.846 & $0.429-1.667$ & 0.628 \\
\hline Low & 0.385 & & & & & \\
\hline \multicolumn{7}{|l|}{ High } \\
\hline NLR & 1 & $1.519-80.07$ & 0.018 & 2.816 & $0.318-24.966$ & 0.352 \\
\hline Low & 11.02 & & & & & \\
\hline \multicolumn{7}{|l|}{ High } \\
\hline PLR & 1 & $1.339-3.908$ & 0.002 & 0.922 & $0.483-1.762$ & 0.806 \\
\hline Low & 2.287 & & & & & \\
\hline \multicolumn{7}{|l|}{ High } \\
\hline SII & 1 & $2.250-17.759$ & $<0.001$ & 3.134 & $0.974-10.087$ & 0.055 \\
\hline Low & 6.322 & & & & & \\
\hline High & & & & & & \\
\hline
\end{tabular}

\section{Discussion}

It is well-known that NLR, PLR, LCR and SIl are important factors in the systemic inflammatory response, and $\mathrm{PNI}$ is an important indicator to evaluate both the nutritional and immune status of patients. A large number of studies have shown that inflammatory and nutritional indicators in the peripheral blood are significantly correlated with the prognosis of breast cancer, rectal cancer and other tumors.LLCR and HPNI have certain effects on the assessment and prediction of the survival rate and postoperative management ${ }^{[7,8,10,11]}$. However, the effect of preoperative LCR and PNI on the prognosis of patients with osteosarcoma is not yet clear, and there are few relevant reports. In this study, preoperative peripheral 
blood inflammatory and nutritional indicators were used to evaluate the prognosis of patients with osteosarcoma in order to obtain a comprehensive and objective predictor and provide relevant theoretical basis for timely treatment. We detected and analyzed the preoperative peripheral blood count and albumin levels of all patients with osteosarcoma, and finally found that LCR and PNI were important factors affecting the postoperative survival rate of patients with osteosarcoma, among which LCR and tumor stage were independent predictors of prognosis.

There is increasing evidence that systemic immune inflammation and nutrition are related to clinical indicators and prognosis of tumor patients ${ }^{[12-14]}$. Our study found that LLCR and LPNI were related to late Enneking stage and more prone to tumor metastasis, which was the same as previous studies. Inflammatory responses can lead to increased neutrophils, thrombocytopenia, lymphocytopenia, elevated C-reactive protein, and decreased serum albumin. As a leukocyte subgroup, both neutrophils and platelets can promote tumorigenesis, proliferation and metastasis by secreting chemokines and vascular endothelial factors, such as vascular endothelial growth factor (VEGF) $[4,15,16]$. The rapid increase in CRP is mainly related to pro-inflammatory factors, such as interleukin (IL)-1, TNF-a, IL-6 and IL-8, and these pro-inflammatory factors regulate cell proliferation and angiogenesis by activating intracellular pathways, such as the pathway mediated by the nuclear factor NF-kB that activates B cells, thereby promoting the development and metastasis of malignant tumors ${ }^{[17-19]}$. Serum albumin, which is produced by the liver, is an important nutritional indicator of the body and plays an important role in improving the body's immunity, suppressing the inflammatory response and anti-tumor effects ${ }^{[20]}$. However, an increase of CRP is often accompanied by a decrease of serum albumin concentration, an increase of tissue consumption, and the continuous loss of body weight, which leads to malnutrition of the body and further aggravation of the disease ${ }^{[21,22]}$. In contrast, lymphocytes are crucial in the body's immunity. During the development of tumor cells, lymphocytes suppress tumor growth and invasion by secreting cytokines such as TNF-a and interferon. Therefore, lymphocytosis often indicates that the host's immune response to tumor is impaired ${ }^{[23-25]}$.In summary, patients with LLCR and LPNI who had an immunosuppressed state and malnutrition had shorter OS time. In addition, our results suggest that patients with LLCR may have HNLR, HPLR and HSII, and the degree of inflammation in vivo may be more severe. At present, it has been reported that systemic inflammatory markers such as preoperative LCR, PNI, NLR, PLR and SIl can be independent predictors of prognosis of gastric cancer, colorectal cancer, lung cancer and osteosarcoma $^{[26-29]}$. As the most prevalent malignant bone tumor, the prognostic factors of osteosarcoma, such as local recurrence, metastasis, tumor stage and pathological fracture, have been reported in a large number of clinical studies. ${ }^{[1,28,30-32]}$. However, there are few studies on the correlation between preoperative LCR and PNI and the prognosis of patients with osteosarcoma. In this paper, the relationship between preoperative LCR, PNI, NLR, PLR and SII and the postoperative OS rate of 101 patients with osteosarcoma was analyzed to explore the important factors affecting the postoperative OS of patients with osteosarcoma. Univariate analysis by the Cox hazard regression model showed that tumor stage $(P<0.001)$, metastasis $(P<0.001)$, LCR $(P<0.001)$, PNI $(P=0.001)$, NLR $(P=0.018), P L R(P=$ $0.002)$ and $S I I(P<0.001)$ were significantly associated with postoperative OS in patients with osteosarcoma. However, after multivariate analysis by the Cox hazard regression model, only tumor stage 
$(\mathrm{HR}, 2.717 ; 95 \% \mathrm{Cl}, 1.067-6.919 ; \mathrm{P}=0.036)$ and preoperative LCR $(\mathrm{HR}, 0.401 ; 95 \% \mathrm{Cl}, 0.199-0.807 ; \mathrm{P}=0.01)$ were independent prognostic factors for OS after surgery. However, Huang et al. ${ }^{\left[{ }^{[2]}\right.}$ considered that PNI and SII were also independent prognostic factors for patients with osteosarcoma, which was different from the results of this study. These differences may be due to the small sample size and heterogeneity of the study population. For example, of the 126 patients with osteosarcoma included in the study by Huang et al, 31.7\% received neoadjuvant chemotherapy. In our study according to the results of the univariate analysis, NLR, PLR, SII, PNI and metastasis were the important factors influencing the postoperative OS in patients with osteosarcoma, but by the multivariate Cox hazards regression model analysis to eliminate confounding factors, the effect of the above factors on the postoperative OS of patients with osteosarcoma decreased obviously.

There are differences in research results related to osteosarcoma in recent years ${ }^{[1,28,30-32]}$. The reasons for this result may be as follows: 1 . the sample size included in this study is small, 2 . The included indicators are too few, 3. the mutual influence of different indicators. There are some limitations to our study. First, the number of cases included in this study is small, with only 101 cases. Second, our study is a single-center retrospective study, which may have a large selection bias, which needs to be further confirmed by a multicenter, large sample size study or prospective study. In addition, there are many factors affecting the prognosis of osteosarcoma, and still need more studies to determine the correlation between different influencing factors. It is still necessary to further explore the relationship between systemic inflammation and nutritional immunity and the prognosis, recurrence and metastasis of osteosarcoma.

\section{Conclusion}

The higher preoperative LCR is strongly associated with the longer survival time in patients with osteosarcoma. Tumor staging and preoperative LCR may be important parameters for the prognosis of osteosarcoma.

\section{Declarations}

\section{Acknowledgements}

Not applicable.

\section{Authors' contributions}

YL and JLH contributed to the study concepts. SYJ and HJT contributed to the study design. XTL and WFL contributed to the data acquisition. XYH contributed to the quality control of data. SJL and FWY contributed to the data analysis and interpretation. $\mathrm{XYH}$ and SJL contributed to the statistical analysis. QJW and YL reviewed the manuscript. All authors read and approved the final manuscript.

\section{Founding}


The study was partly supported by Natural Science Foundation of Guangxi Province (2019JJA140408), Youth Science and Technology Project of the First Affiliated Hospital of Guangxi Medical University (201903038), Guangxi Health Department self-raised funds project (Z20170424), and Science and Technology Base and Talent Project in Qingxiu District, Nanning City (2020068).

\section{Availability of data and materials}

All data used in the study are available from the corresponding authors.

\section{Ethics approval and consent to participate}

This study was approved by the Ethics Committee of the First Affiliated

Hospital of Guangxi Medical University.

\section{Consent for publication}

All participants consented to the publication.

\section{Competing interests}

The authors declare that they have no competing interests.

\section{Author details}

${ }^{1}$ General Medicine Ward of the First Affiliated Hospital of Guangxi Medical University, Nanning, Guangxi, China. ${ }^{2}$ Department of Orthopaedics, the Minzu Affiliated Hospital of Guangxi Medical University, Nanning, Guangxi, China. ${ }^{3}$ Department of Pharmacy,

First Affiliated Hospital of Guangxi Medical University, Nanning, Guangxi, China. ${ }^{4}$ Department of Orthopaedics, First Affiliated Hospital of Guangxi Medical University, Nanning, Guangxi, China. ${ }^{5}$ Spinal Surgery Department, First Affiliated Hospital of Guangxi University of Traditional Chinese Medicine.

${ }^{6}$ Department of Bone and Soft Tissue Surgery, Guangxi Medical University Cancer Hospital.

\section{References}

1. Yang Q, Chen T, Yao Z, et al. Prognostic value of pre-treatment Naples prognostic score (NPS) in patients with osteosarcoma, World Journal of Surgical Oncology. 2020. 18(1).

2. Liu M, Yang P, Mao G, et al. Long non-coding RNA MALAT1 as a valuable biomarker for prognosis in osteosarcoma: A systematic review and meta-analysis. 72. London: International Journal of Surgery; 2019.

3. Xu S, Gong Y, Yin Y, et al. The multiple function of long noncoding RNAs in osteosarcoma progression, drug resistance and prognosis. Biomed Pharmacother. 2020;127:110141. 
4. Colotta F, Allavena P, Sica A, et al. Cancer-related inflammation, the seventh hallmark of cancer: links to genetic instability, Carcinogenesis. 2009.

5. Alberto M. Paola, et al. Cancer-related inflammation, Nature. 2008.

6. Hirahara T, Arigami T, Yanagita S, et al. Combined neutrophil-lymphocyte ratio and plateletlymphocyte ratio predicts chemotherapy response and prognosis in patients with advanced gastric cancer. BMC Cancer. 2019;19(1):672.

7. Shinsuke S, Takashi A, Koji O, et al. Comprehensive Comparative Analysis of Prognostic Value of Systemic Inflammatory Biomarkers for Patients with Stage II/III Colon Cancer, Annals of Surgical Oncology. 2020. 27(3):844-852.

8. Yoshinaga O, Yuji T, Akira Y, et al. Lymphocyte-C-reactive Protein Ratio as Promising New Marker for Predicting Surgical and Oncological Outcomes in Colorectal Cancer, Annals of Surgery. 2019.

9. Pointer DT, Roife D, Powers BD, et al. Neutrophil to lymphocyte ratio is predictive of patient survival after resection of pancreatic adenocarcinoma. HPB. 2019;21(1):128.

10. Okugawa Y, Toiyama Y, Fujikawa $\mathrm{H}$, et al. Prognostic Potential of Lymphocyte-C-Reactive Protein Ratio in Patients with Rectal Cancer Receiving Preoperative Chemoradiotherapy, Journal of Gastrointestinal Surgery. 2020(1).

11. He H, Guo W, Song P, et al. Preoperative systemic immune-inflammation index and prognostic nutritional index predict prognosis of patients with pulmonary neuroendocrine tumors after surgical resection. Annals of Translational Medicine. 2020;8(10):630-0.

12. Wang Y, Zhu Z, Li C, et al. Prognostic significance of preoperative albumin-to-globulin ratio and prognostic nutritional index combined score in Siewert type 3 adenocarcinoma of esophagogastric junction, Cancer Management and Research. 2019. 11.

13. Carr BI,Guerra $\vee$ Serum albumin levels in relation to tumor parameters in hepatocellular carcinoma patients, Int J Biol Markers. 2017. 32(4).

14. Ikeda $\mathrm{S}$, Yoshioka $\mathrm{H}$, Ikeo $\mathrm{S}$, et al. Serum albumin level as a potential marker for deciding chemotherapy or best supportive care in elderly, advanced non-small cell lung cancer patients with poor performance status. BMC Cancer. 2017;17(1):797.

15. Tecchio C. Neutrophil-derived cytokines involved in physiological and pathological angiogenesis. Chemical Immunology Allergy. 2014;99:123.

16. Mantovani A. Cassatella, et al. Neutrophils in the activation and regulation of innate and adaptive immunity, Nature Reviews Immunology. 2011.

17. Gabay C, Kushner, et al. Acute-Phase Proteins and Other Systemic Responses to Inflammation, New England Journal of Medicine. 1999.

18. Ilhan $\mathrm{N}$, Ilhan $\mathrm{N}$, Ilhan $\mathrm{Y}$, et al. C-reactive protein, procalcitonin, interleukin-6, vascular endothelial growth factor and oxidative metabolites in diagnosis of infection and staging in patients with gastric cancer. World J Gastroenterol. 2004;10(008):1115-20. 
19. Pasqualesansone,Marcu. K IL-6 triggers malignant features in mammospheres from human ductal breast carcinoma and normal mammary gland. Journal of Clinical Investigation. 2007;117(12):3988.

20. Seaton K. Albumin concentration controls cancer. J Natl Med Assoc. 2001;93(12):490-3.

21. Mcmillan DC. The systemic inflammation-based Glasgow Prognostic Score: a decade of experience in patients with cancer. Cancer Treat Rev. 2013;39(5):534-40.

22. Roxburgh CSD. Mcmillan DC Cancer and systemic inflammation: treat the tumour and treat the host. Br J Cancer. 2014;110(6):1409-12.

23. Giorgi UD, Mego M, Scarpi E, et al. Relationship Between Lymphocytopenia and Circulating Tumor Cells as Prognostic Factors for Overall Survival in Metastatic Breast Cancer, Clinical Breast Cancer. 2012. 12(4).

24. Ownby HE, Roi LD, Isenberg RR, et al. Peripheral lymphocyte and eosinophil counts as indicators of prognosis in primary breast cancer. Cancer. 1983;52(1):126-30.

25. Chen L, Yan Y, Zhu L, et al. Systemic immune-inflammation index as a useful prognostic indicator predicts survival in patients with advanced gastric cancer treated with neoadjuvant chemotherapy. Cancer Management Research. 2017;9:849-67.

26. Tiainen S, Rilla K, Hmlinen K, et al. The prognostic and predictive role of the neutrophil-to-lymphocyte ratio and the monocyte-to-lymphocyte ratio in early breast cancer, especially in the HER2 + subtype, Breast Cancer Research and Treatment. 2020:1-10.

27. Li X, Yao Z, Wan Y, et al. Prognostic impact of prognostic nutritional index in advanced (stage IIIB/IV) non-small cell lung cancer patients. Neoplasma. 2019;66(6):971-7.

28. Xin, Huang $\mathrm{H}$, et al. Prognostic value of prognostic nutritional index and systemic immuneinflammation index in patients with osteosarcoma, Journal of Cellular Physiology. 2019.

29. Okugawa $Y$, Toiyama $Y$, Yamamoto $A$, et al. Lymphocyte-to-C-reactive protein ratio and score are clinically feasible nutrition-inflammation markers of outcome in patients with gastric cancer, Clinical Nutrition. 2019.

30. Yong-Jiang L, Kai Y, Min-Xun L, et al. Prognostic value of the C-reactive protein to albumin ratio: a novel inflammation-based prognostic indicator in osteosarcoma. Oncotargets Therapy. 2017;10:5255-61.

31. Liu B, Huang Y, Sun Y, et al. Prognostic value of inflammation-based scores in patients with osteosarcoma. Sci Rep. 2016;6(1):39862.

32. Liu T, Fang X-C, Ding Z, et al. Pre-operative lymphocyte-to-monocyte ratio as a predictor of overall survival in patients suffering from osteosarcoma. Febs Open Bio. 2015;5(1):682-7.

\section{Figures}




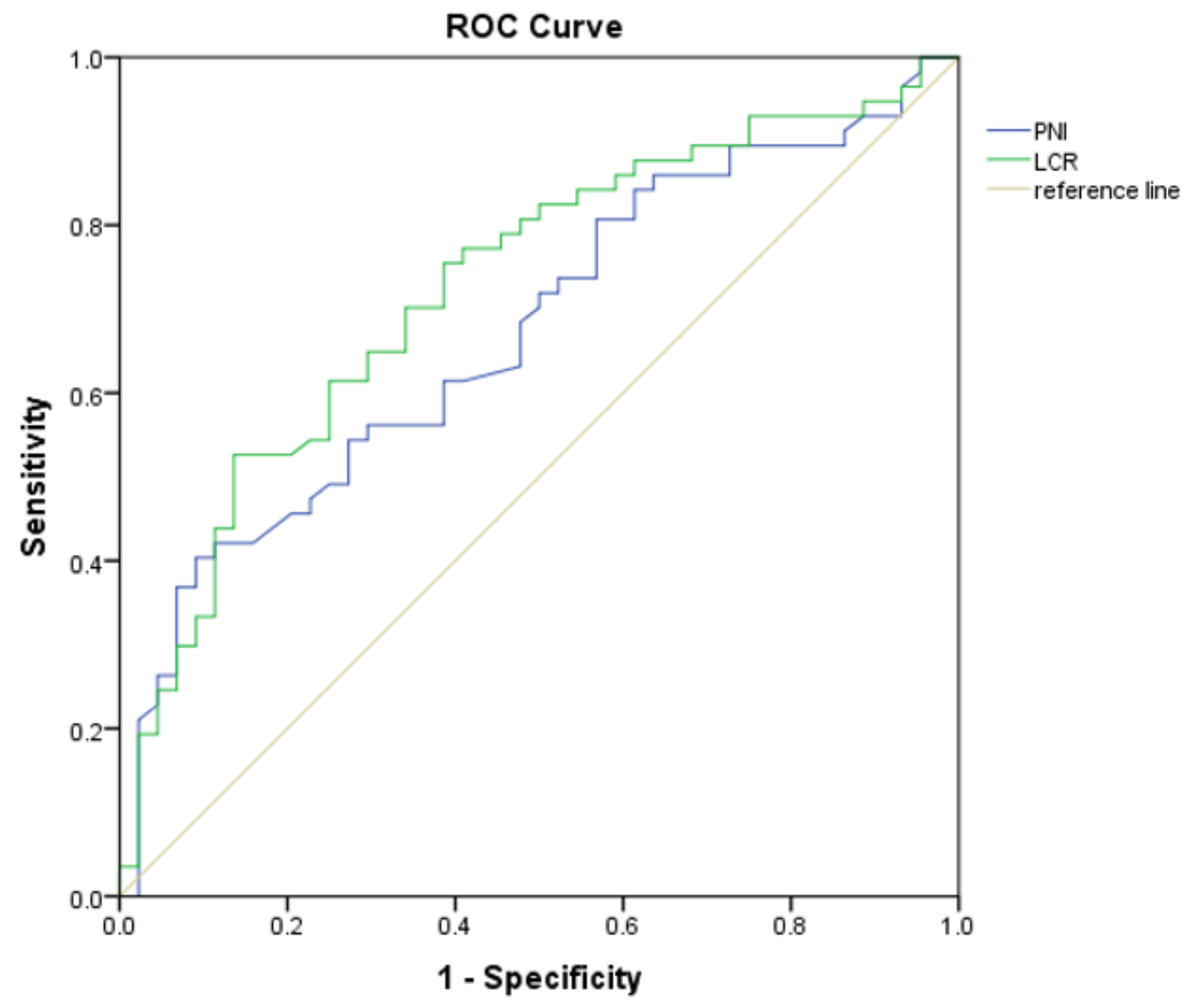

Figure 1

Receiver operating characteristic curves for pretreatment LCR and PNI based on OS. OS: overall survival; LCR: lymphocyte count and C-reactive protein ratio; PNI: prognostic nutritional index. 

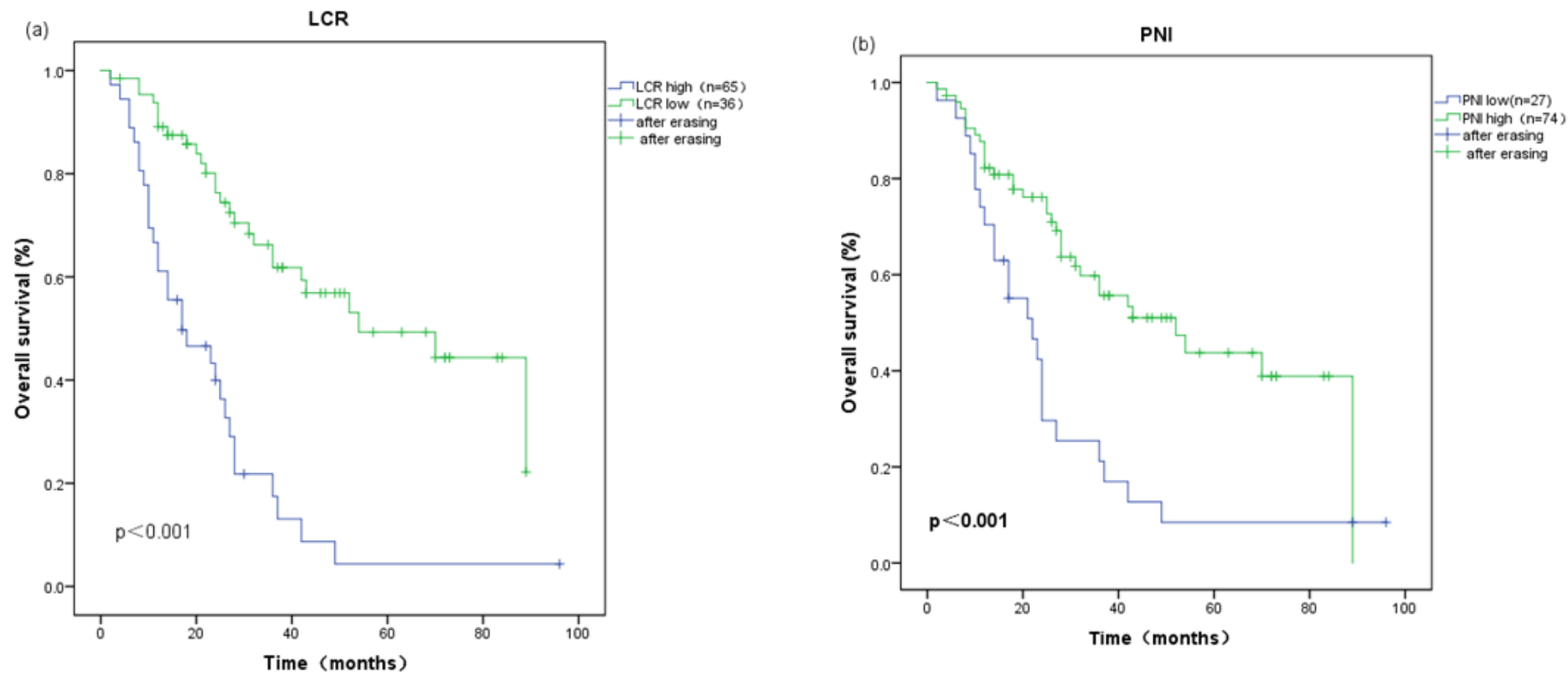

Figure 2

Kaplan-Meier survival curves for OS according to LCR and PNI in osteosarcoma patients. (a) OS of patients in the LLCR group was significantly worse than those in the HLCR group $(p<0.001)$. (b) OS of patients in the HPNI group was significantly better than those in the LPNI group $(p<0.001)$. 